\title{
Qualitative Human Chorionic GonadotropinTesting in Vaginal Fluid for Diagnosis of Preterm Premature Rupture of Membrane
}

\author{
Reeti Mehra, Anju Huria, Beena Mahaur \\ Dept of Obstetrics \& Gynaecology, \\ Government Medical College \& Hospital, Chandigarh, India
}

\begin{abstract}
The diagnosis of preterm premature rupture of membrane is usually done by history, visible pooling of fluid on speculum and positive litmus test. HCG in cervical secretions is found in High concentrations till 20 weeks period of gestation (POG) but after 20 weeks, it remains at a stable level, where median levels were between 5.6 and $7.1 \mathrm{mlU} / \mathrm{ml}$ in various studies.

This study was performed to determine whether a qualitative human chorionic gonadotropin (hCG) test using vaginal washings is a useful indicator of preterm premature rupture of membranes (PROM).

Methods: A prospective analysis of qualitative hCG testing on vaginal washing in 29 patients with known PROM and in 20 pregnant women with intact membranes was done. Patients with singleton pregnancies between 28 - 34 weeks were included. A commercially available one step qualitative slide pregnancy test kit with a threshold of $25 \mathrm{mlU} / \mathrm{ml}$ was used. Capsule: Vaginal fluid was tested by commercially available (BHCG) kits to diagnose preterm, premature rupture of membranes. Statistical analysis Data was analysed with $t$ test, $x^{2}$ and Fisher exact test.

Results: Forty nine subjects were enrolled in the study with 29 in the PROM group and 20 in the control group. The two groups were similar with respect to parity and period of gestation. The hCG test was positive in $19(65 \%)$ of the PROM group and $11(55 \%)$ of the control group.

(Sensitivity 65.5\%, specificity 45\%, PPV 63.3\%, NPV 47.36\%). The overall diagnostic accuracy of the test was $57.14 \%$.

Conclusion: Qualitative hCG testing of vaginal washings does not appear to be a useful predictor of preterm premature rupture of membranes.

Key Words: Preterm Premature rupture of membrances (PROM), human chorionic gonadotropin (hCG), diagnosis
\end{abstract}

\section{Introduction}

Preterm birth remains the leading cause of neonatal morbidity and mortality throughout the world despite advances in perinatal and neonatal care. Preterm premature rupture of membranes (PROM) has be estimated to affect $3 \%$ to $4.5 \%$ of all deliveries and 32 to $40 \%$ of preterm deliveries. ${ }^{1}$ Perinatal morbidity and mortality associated with PROM are related both independently and by increased rates of preterm birth. The independent relationship with perinatal complications has been illustrated by Arias and Tomich ${ }^{2}$ who have prospectively shown higher rates of severe neonatal morbidity in pregnancies complicated by PROM than in those caused by idiopathic preterm labour (27\% versus $15.1 \%, \mathrm{p}<0.02)$. Inability to correctly diagnose PROM may delay the definitive treatment in the form of antenatal steroids and antimicrobial therapy. Incorrect diagnosis may also lead to unnecessary intervention and iatrogenic prematurity.

\section{Correspondence}

Dr.Reeti Mehra MD

Assoc Prof Deptt. of Obstetrics \& Gynaecology,

Government Medical College \& Hospital, GMCH-32, Chandigarh-160 030

Residential House No 1501, Sector 33 D, Chandigarh.

Phone No. 09876521581 Mobile No. 9646121581

Fax No.0172-2609360 \& 2608488, E.mail:drreetidatta@yahoo.co.in 
The history of the patient, direct visualization of amniotic fluid, litmus test, ferning are the currently available methods used. All these simple tests have their own false positive and false negative results and can make the diagnosis difficult in some situations.

Vaginal fluid creatinine, alpha fetoprotein, prolactin, fetal fibronectin, insulin like growth factor, and even thyroid hormones have been evaluated earlier but these are time consuming, expensive, not readily available and have limited practical use.

Human chorionic gonadotropin is produced by trophoblastic tissue and is present in serum, urine and amniotic fluid. In a study of Bernstein et $\mathrm{al}^{3} \mathrm{hCG}$ in cervical secretions is found in high concentrations till 20 weeks period of gestation (POG) but after 20 weeks, it remains at a stable level, where median levels were between 5.6 and $7.1 \mathrm{mIU} / \mathrm{ml}$. Anai et $\mathrm{al}^{4}$ compared the levels of hCG in patients with and without PROM. The median level of vaginal fluid hCG of normal pregnant women was $37.9,9.5$ and $6.3 \mathrm{mIU} / \mathrm{ml}$ during $1^{\text {st }}, 2^{\text {nd }}$ and $3^{\text {rd }}$ trimesters respectively as against $420.6 \mathrm{mIU} / \mathrm{ml}$ in women with confirmed PROM. The present study was carried out to see if qualitative bHCG test by commercially available ELISA pregnancy test kits could accurately diagnose PROM. The test is easily available, inexpensive, easy to perform and the result is available immediately.

\section{Methods}

A prospective study of pregnant women between 28 and 34 weeks period of gestation was conducted between January 2006 and June 2006. It was approved by the Ethical Committee of the institution.

The study group consisted of women with confirmed rupture of amniotic membranes documented by history, visualized amniotic fluid pooling on speculum and positive litmus test. All patients were between 28 and 34 weeks period of gestation confirmed by ultrasonograpy. The control group consisted were pregnant women with intact amniotic membranes coming to the antenatal outpatient department for routine check up between 28 - 34 weeks period of gestation

Patients with vaginal bleeding, multiple pregnancy or congenital malformation were excluded in both groups. This gestational age range was chosen since these patients might benefit from conservative management and antibiotic prophylaxis if PROM was proven. All patients were sure of dates and had dates confirmed by ultrasonography.

The vaginal pooled liquor was taken directly for conducting the $\mathrm{b}$ hCG testing or posterior vaginal fornix was irrigated with $3-5 \mathrm{ml}$ of saline and then with the aspirated washings the test was conducted. The one step pregnancy test kit with a threshold of $25 \mathrm{mIU} / \mathrm{ml}$ was used.

The test was interpreted as positive or negative. All samples were obtained and confirmed by senior residents (post MD) working in the department.

The 2 groups were compared for parity, gestational age and hCG test result. $\mathrm{c}^{2}$ and Fischer exact test was used along with paired t test. All $\mathrm{P}<.05$ were considered significant. Sensitivity, specificity, predictive values and overall test efficiency was calculated to the basis of results of hCG test.

\section{Results}

Forty-nine subjects were sampled with 29 in the PROM group and 20 in the control group. Both groups were similar with respect to maternal age and gestational age (Table I). The PROM group was sampled at median gestational age of 33.07 weeks and in the control group, the median gestational age was 33.6 weeks.

\section{Table 1. Population characteristics of the study}

\begin{tabular}{llll}
\hline & Cases $(\mathbf{n}=\mathbf{2 9})$ & Control groups $(\mathbf{n}=\mathbf{2 0})$ & Pvalue \\
\hline Maternal age & $23.79 \pm 2.37$ & $23.95 \pm 2.21$ & $\mathrm{P}>0.20$ (NS) \\
EGA (weeks) & $33.07 \pm 1.94$ & $33.6 \pm 1.64$ & $\mathrm{P}>0.20$ (NS) \\
Parity & $1.62 \pm 0.62$ & $1.35 \pm 0.59$ & $\mathrm{P}>0.10$ (NS) \\
Nulliparous & $13(44.8 \%)$ & $12(60 \%)$ & $\mathrm{P}>0.20$ \\
Multiparous & $16(55.2 \%)$ & $8(40 \%)$ & $\chi^{2}=0.295$ \\
\hline
\end{tabular}

Data are presented as means $\pm S D$. 
Table 2. Human chorionic gonadotropin (HCG) results

\begin{tabular}{llll}
\hline & PROM $(\mathbf{n}=\mathbf{2 9})$ & Control $(\mathbf{n}=\mathbf{2 0})$ & \\
\hline HCG + & 19 & 11 & PPV 63.3\% \\
HCG- & 10 & 09 & NPV $47.36 \%$ \\
\hline
\end{tabular}

In the PROM patients only 19 out of 28 had a positive hCG test resulting in the sensitivity of $65.5 \%$. Conversely 9 out of 20 control patients had a negative hCG test for a specificity of only $45 \%$. Our study demonstrated that a positive hCG test had a positive predictive value (PPV) of $63.3 \%$ and negative predictive value (NPV) of $47.36 \%$ (Table II). The overall diagnostic accuracy of the test was only $57.14 \%$.

\section{Discussion}

Establishing the accurate diagnosis of PROM may result in significant cost saving due to unnecessary admissions, treatment and also unnecessary transfers to tertiary care centres with neonatal intensive care unit. It would prevent iatrogenic prematurity in certain cases and by timely administration of costicosteroids would reduce the respiratory distress rates in confirmed cases.

The study was conducted keeping in mind the various studies where levels of hCG in normal pregnant women were compared with those of women with confirmed PROM. ${ }^{3-5}$ Based on the above studies, we conducted our study since qualitative bhCG is a simple, non invasive, cost-effective and easy to perform test and would make a tremendous impact on the diagnosis and treatment for these patients.

On review of literature we could find only one study by Amy L Cooper et $\mathrm{al}^{6}$ using qualitative bhCG for diagnosis of PROM. They had reported a PPV of $95 \%$ and NVP of $84 \%$ thus establishing a good correlation. However, we were unable to demonstrate a good sensitivity or specificity.

The conclusion of our study was in accordance with studies by Kim YH et $\mathrm{al}^{7}$ and Li HY et al. ${ }^{8}$ They also reported median range of vaginal fluid bhCG as 3.6 in normal patients but gave a range from $0.09-30.52 \mathrm{mIU} /$ $\mathrm{ml}$ as against $512(26.95-3507.20) \mathrm{mIU} / \mathrm{ml}$ in those with confirmed PROM. From the receiver operating characteristic curve, $39.8 \mathrm{mIU} / \mathrm{ml}$ was set as a cut off value in their study. Li HY et al ${ }^{[8]}$ gave median levels of vaginal fluid hCG of normal pregnant women as $35 \mathrm{mIU}$ and those with confirmed PROM as $478 \mathrm{mIU} / \mathrm{ml}$. More recently, Shahin $\mathrm{M}$ et al ${ }^{9}$ also has reported a PPV of $75 \%$ and NPV of $85 \%$ of bhCG as a marker for PROM and has concluded that it is less reliable as compared to alpha-fetoprotein for diagnosis of PROM.
Bernstein et $a{ }^{3}$ proposed that as a result of inflammatory process that proceeds the onset of labour, hCG escapes from mat serum to cervical secretions. They reported a cut off of bhCG $>50 \mathrm{mIU} / \mathrm{ml}$ in prediction of preterm vaginal delivery. A. Gurbuz et al ${ }^{10}$ also concluded that cervical hCG is rewarding for diagnosis of preterm labour and recommended treatment for prevention of preterm delivery may be started at cervical bhCG $>32 \mathrm{mIU} / \mathrm{ml}$.

We concluded that the one step kit with a threshold of $25 \mathrm{mIU} / \mathrm{ml}$ is not a reliable testing means for confirming or ruling out PROM. bhCG in vaginocervical secretions may by increased in patients with threatened preterm labour and any vaginal bleeding or show may further skew the results. Also in various previous studies clearly the bHCG may range from $0.09-35 \mathrm{mIU} / \mathrm{ml}$ in normal pregnant patients, though median values are reported low, which explains the results of our study.

Using a qualitative test which detects bhCG $50 \mathrm{mIU} / \mathrm{ml}$ may have a better sensitivity but are not easily available and are thus less practical. Though our sample size was small, we did not find the one step pregnancy test kits with threshold of bhCG $25 \mathrm{mIU} / \mathrm{ml}$ useful to diagnose PROM.

Conclusion: Qualitative bhCG testing of vaginal washings does not appear to be a useful predictor of preterm premature rupture of membranes.

\section{References}

1. Mercer BM, Goldenberg RL, Meis PJ et al. The preterm prediction study: Prediction of preterm premature rupture of membranes through clinical findings and ancillary testing. Am J Obstet Gynecol 2000; 183: 738.

2. Arias F, Tomich P: Etiology and outcome of low birth weight and preterm infants. Obstet Gynecol 1982, 60: 277..

3. Bernstein PS, Stern R, Lin N, Furgiuele J, Krmen A, Comerford Freda $\mathrm{M}$ et al. Bhuman chorionic gonadotropin in cervicovaginal secretions as a predictor of preterm delivery. Am J Obstet Gynecol 1998; 79: 870-3.

4. Anai T, Tanaka Y, Hirota Y, Miyakawa I. Vaginal fluid hCG levels for detecting premature rupture of membranes. Obstet Gynecol 1997; 89: 261-4. 
5. Esim E, Turan C, Unal O, Dansuk R, Cengizglu B. Diagnosis of premature rupture of membranes by identification of beta - hCG in vaginal washing fluid. Eur J Obstet Gynecol Reprod Biol 2003; 107(1):37-40.

6. Amy L. Cooper, Stephen T. Vermillion, David E. Soper. Qualitative human chorionicgonadotropin testing of cervicovaginal washings for the detection of preterm premature rupture of membranes. Am J Obstet Gynae 2004; 191: 593-7.

7. Kim YH, Park YW, Kwon HS, Kwon JY, Kim BJ. Vaginal fluid bets human chorionic gonadotropin level in the diagnosis of premature rupture of membranes. Acts Obstet Gynecol Scand 2005; 84(8): 802-5.
8. Li HY, Chang TS. Vaginal fluid creatinine, human chorionic gonadotropin and alphafetoprotein levels for detecting premature rupture of membranes. Zhonghua Yi Xue Za Zhi (Taipei) 2000; 63(9): 689-90.

9. Shahin M, Raslan H. Comparative study of three amniotic fluid markers in premature rupture of membranes: prolactin, beta subunit of human chorionic gonadotropin and alphafetoprotein. Gynecol Obstet Invest 2007; 63(4): 195-9.

10. GurbuzA, Karateke A, Ozturkmen M, Kaliaca C. Human chorionic gonadotropin assay in cervical secretion for accurate diagnosis of preterm labour. International Journal of Gynaecology and Obstetrics 2004; 85: 132-8. 